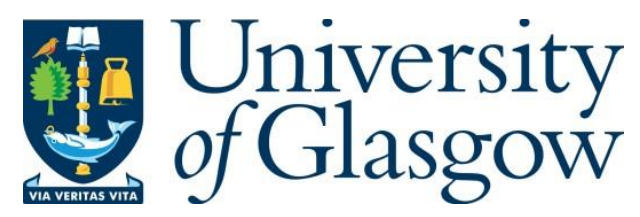

Chen, J. and Ewald, C.-O. (2017) Pricing commodity futures options in the Schwartz multi factor model with stochastic volatility: An asymptotic method. International Review of Financial Analysis, 52, pp. 144-151.

There may be differences between this version and the published version. You are advised to consult the publisher's version if you wish to cite from it.

http://eprints.gla.ac.uk/143724/

Deposited on: 18 July 2017

Enlighten - Research publications by members of the University of Glasgow http://eprints.gla.ac.uk 


\title{
Pricing Commodity Futures Options in the Schwartz Multi Factor Model with Stochastic Volatility: An Asymptotic Method
}

\author{
Jilong Chen*and Christian Ewald ${ }^{\dagger}$
}

March 22, 2017

\begin{abstract}
In this paper we investigate the applicability of the asymptotic approach developed in Fouque et al. (2000) for pricing commodity futures options in a Schwartz (1997) multi factor model, featuring both stochastic convenience yield and stochastic volatility. We show that the zero order term in the expansion coincides with the Schwartz (1997) two factor term, with expected long-term volatility replacing the constant volatility term, and provide an explicit expression for the first order correction term. Using empirical data from the natural gas futures market, we demonstrate that a significantly better calibration can be achieved by involving the correction term as compared to the standard Schwartz (1997) two factor expression. This improvement comes at virtually no extra effort.
\end{abstract}

Keywords: Commodities; Derivatives; Stochastic Volatility; Stochastic Convenience Yield

*Postal address: International Institute for Financial Studies and RCFMRP, Jiangxi University of Finance and Economics,Nanchang, 330013, Jiangxi Province, China. e-mail: cjlhk1989@hotmail.com

${ }^{\dagger}$ Postal address: University of Glasgow, Adam Smith Business School, Department of Economics, Gilbert Scott Building, Glasgow, G12 8QQ, United Kingdom. Corresponding author e-mail: christian.ewald@glasgow.ac.uk, Tel.: +44(0)1413305354 


\section{Introduction}

A futures option is an option contract in which the underlying is a single futures contract. The buyer of a futures option has the right, but not the obligation, to enter into a futures contract at a certain futures price at a certain date. The seller must take the opposite position in the futures contract when the buyer exercises this right. Specifically, a futures call option is the right to enter into a long futures contract at a certain futures price; a futures put option is the right to enter into a short futures contract at a certain futures price (Hull (2006)). Predominantly, futures options are American style options, which can be exercised at any time during the life of the contract. However, for a number of energy commodities, including crude oil and natural gas, futures options are also available as European style options. It is important to note that the underlying of a futures option is the futures price, and not the commodity itself. Therefore, the futures option's price is tied to the futures price, and only indirectly to the commodity price. Futures prices for contracts close to maturity track the corresponding commodity price rather closely.

The convenience yield of a commodity is the benefit obtained from holding the spot commodity instead of the futures contracts. This is important when pricing futures contracts, see Brennan (1991). Gibson and Schwartz (1990) found that a constant convenience yield does not work well for pricing futures contracts. Miltersen and Schwartz (1998) and Schwartz (1997) have developed one-, two- and three-factor models to price commodity futures contracts and futures options with stochastic convenience yields and interest rates. Their results confirmed that constant convenience yield is a rather ill-advised assumption and that a stochastic convenience yield is far better able to fit the different observed shapes of the forward curves. Hilliard and Reis (1998) assumed additionally that the underlying spot price next to a stochastic convenience yield also features a Poisson jump term and show that the relevant option pricing formula is a weighted sum over the corresponding Schwartz (1997) two factor expressions, similar as Merton (1976).

Stochastic volatility models have become more and more popular for derivatives pricing and hedging, especially since the 1987 market crash. In the Black-Scholes framework, the log returns of assets are assumed to follow a normal distribution and many other models also share this assumption, including the celebrated Schwartz (1997) two factor model. This has the advantage that it often leads to a closed form solution for derivatives prices, but empirical studies strongly contradict the normality assumption of re- 
turns. Generally, empirical log returns of equities, currencies and commodities have higher peaks and fatter tails, which is indicative of a distribution with differing variances, see Gatheral (2011), Cassese and Guidolin (2006) and Chiarella et al. (2015). Options, in terms of implied volatilities which are anything but flat, provide further evidence that underlyings are not lognormal. Recently, Trolle and Schwartz (2009) provided strong evidence that for both futures and options on Brent crude oil it is necessary to consider stochastic volatility when pricing derivatives. In difference to the classical Schwartz (1997) approach, they model the entire forward cost of carry curve in a fashion similar to the Heath-Jarrow-Morton approach for interest rates, with added stochastic volatility to both spot and cost of carry. However, they do not derive an explicit expression for the price of an option in their model. Rauch et al. (2013) discuss the issue of volatility and mean reversion in the pricing of commodity derivatives and calibrate their model to market data from a range of commodities. However, in difference to our paper, they work with constant convenience yield and the assumption that volatility is a function of the commodity price only and not affected by exogenous shocks. In consequence their model is one dimensional, while our model is three dimensional.

In fact, it is very difficult to find closed form solutions for option prices in models with stochastic volatility, especially when convenience yield is also assumed to be stochastic. For constant convenience or dividend yield, Fouque et al. (2000) assumed that the spot volatility follows a mean reverting Ornstein-Uhlenbeck (OU) process and presented an asymptotic expansion to calculate European derivatives prices. The zero order term in this expansion corresponds to the classical Black-Scholes term, where the constant spot volatility is replaced by the long term average volatility. This technique has been extended to cover other volatility dynamics, including Heston volatility, in Ting and Ewald (2013) as well as for real options and optimal exercise timing in Ting et al. (2013) and Agarwal et al. (2016). Recently, Barsotti and Pontier (2016) used the asymptotic approach to study the optimal capital structure in a Merton structural firm model with stochastic volatility. Zaevski et al. (2014) derive an analytical formula for pricing a European call option in a Heston model with tempered stable Lévy jumps but do not consider stochastic convenience yield.

In the current paper we take on the Schwartz (1997) multi-factor model with stochastic convenience yield, which is a benchmark in the commodity literature, and add stochastic volatility of OU type. This model is a good candidate for the applicability of Fouque et al. (2000)'s methodology, as in the constant volatility version it admits closed form expression for the price of 
European calls and puts which are of a modified Black-Scholes type. In fact, we are able to show that the zero order term in our expansion coincides with the classical expression derived in Hilliard and Reis (1998) for the Schwartz (1997) two factor model with constant volatility and in addition provide an explicit expression for the first order correction term. This correction term is easy to evaluate and in fact the combined expression, consisting of zero order term and correction term, is no harder to evaluate than the Hilliard and Reis (1998) formula. We then demonstrate by looking at data for European call options on natural gas, that by taking account of the correction term, a significantly better fit can be obtained.

The remainder of this paper is organized as follows: Section 2 briefly covers our three factor model which includes spot price, convenience yield and stochastic spot volatility. In section 3 , we derive the asymptotic expression for futures options in terms of the spot price as underlying. In section 4, we briefly review the pricing of European commodity options under the Schwartz (1997) two factor model. In section 5 we show how the asymptotic solution for futures options can also be expressed in terms of futures price as the underlying, which is closer in line with actual trading. In section 6 and section 7 , we examine the asymptotic solution for futures options through simulated and historical data and use the Schwartz (1997) two factor model for comparison. Section 8 summarizes the main conclusions.

\section{Schwartz' Model with Stochastic Volatility}

Our model consists of the three factors - - spot price $S_{t}$, convenience yield $y_{t}$ and spot volatility $V_{t}$. For pricing futures and futures options their dynamics under a chosen risk-neutral measure $\mathbb{Q}$ is relevant. We assume that under $\mathbb{Q}$ we have that

$$
\begin{aligned}
& d S_{t}=\left(r-y_{t}\right) S_{t} d_{t}+f\left(V_{t}\right) S_{t} d W_{1} \\
& d y_{t}=\left(\kappa_{1}\left(\alpha-y_{t}\right)-\lambda\right) d t+\sigma_{c} d W_{2} \\
& d V_{t}=\left(\kappa_{2}\left(m-V_{t}\right)-\Lambda\left(V_{t}\right)\right) d t+\beta d W_{3},
\end{aligned}
$$

with $W_{i}$ correlated Brownian motions, s.t. $d W_{i} \cdot d W_{j}=\rho_{i j} d_{t}$ and $f(V)$ is a suitable and sufficiently smooth function. ${ }^{1}$ The parameter $\lambda$ represents the

\footnotetext{
${ }^{1}$ Volatility, at least under the real world measure, the follows an Ornstein-Uhlenbeck process. This process is also the focus of Fouque et al. (2000). It has the disadvantage of taking negative values, even though the instantaneous variance of returns $\frac{d S_{t} d S_{t}}{S_{t}^{2}}=$ $f\left(V_{t}\right)^{2} d t$ is positive no matter what. The techniques of Fouque et al. (2000) have been extended to cover volatility of Cox-Ingersoll-Ross (CIR) or Heston type in Ting and Ewald
} 
market price of convenience yield risk and

$$
\Lambda(V)=\rho_{13} \frac{\mu-r}{f(V)}+\gamma(V) \sqrt{1-\rho_{13}^{2}},
$$

where $\gamma$ is the market price of volatility risk. We assume that $\gamma(V)$ is a bounded function of $V$ alone. $^{2}$

The key realization in the approach by Fouque et al. (2000) is to consider the rate of mean reversion $\kappa_{2}$ as a large parameter and consequently the parameter $\varepsilon=1 / \kappa_{2}$ as a small parameter and then expand the pricing PDE and solution in terms of orders of $\epsilon$.

Under the Ornstein-Uhlenbeck volatility assumption, the variance of the invariant distribution of $V$, denoted as $v^{2}$, can be expressed as $v^{2}=\beta^{2} /\left(2 \kappa_{2}\right)$; hence, $\beta=\frac{v \sqrt{2}}{\sqrt{\varepsilon}}$. Therefore, under the risk-neutral measure $\mathbb{Q}$, the stochastic differential equation (1) can be rewritten as

$$
\begin{aligned}
& d S_{t}=\left(r-y_{t}\right) S_{t} d_{t}+f\left(V_{t}\right) S_{t} d W_{1} \\
& d y_{t}=\left(\kappa_{1}\left(\alpha-y_{t}\right)-\lambda\right) d t+\sigma_{c} d W_{2} \\
& d V_{t}=\left(\frac{1}{\varepsilon}\left(m-V_{t}\right)-\frac{v \sqrt{2}}{\sqrt{\varepsilon}} \Lambda\left(V_{t}\right)\right) d t+\frac{v \sqrt{2}}{\sqrt{\varepsilon}} d W_{3} .
\end{aligned}
$$

Under no-arbitrage, the value of a contingent claim $P(t, S, y, V)$ with payoff $h(S)$ must satisfy the following partial differential equation and boundary condition:

$$
\begin{gathered}
\frac{\partial P}{\partial t}+\frac{1}{2} f(V)^{2} S^{2} \frac{\partial^{2} P}{\partial S^{2}}+\frac{1}{2} \sigma_{c}^{2} \frac{\partial^{2} P}{\partial y^{2}}+\frac{v^{2}}{\varepsilon} \frac{\partial^{2} P}{\partial V^{2}}+\rho_{12} f(V) S \sigma_{c} \frac{\partial^{2} P}{\partial S \partial y}+\rho_{13} f(V) S \frac{v \sqrt{2}}{\sqrt{\varepsilon}} \frac{\partial^{2} P}{\partial S \partial V}+ \\
\rho_{23} \sigma_{c} \frac{v \sqrt{2}}{\sqrt{\varepsilon}} \frac{\partial^{2} P}{\partial y \partial V}+(r-y) S \frac{\partial P}{\partial S}+\left(\kappa_{1}(\alpha-y)-\lambda\right) \frac{\partial P}{\partial y}+\left[\frac{1}{\varepsilon}(m-V)-\frac{v \sqrt{2}}{\sqrt{\varepsilon}} \Lambda(V)\right] \frac{\partial P}{\partial V}-r P=0
\end{gathered}
$$

and

$$
P(T, S, y, V)=h(S) \text {. }
$$

\subsection{The Operator Notation}

In order to account for terms of order $1 / \varepsilon, 1 / \sqrt{\varepsilon}, 1$ in the partial differential equation (4), we introduce the following convenient notation:

$$
\mathcal{L}_{0}=v^{2} \frac{\partial^{2}}{\partial V^{2}}+(m-V) \frac{\partial}{\partial V}
$$

\footnotetext{
(2013).

${ }^{2}$ For calibration, a suitable parametric form would need to be chosen; often it is chosen as constant or proportional to $V$.
} 


$$
\begin{aligned}
& \mathcal{L}_{1}=\sqrt{2} v \rho_{13} f(V) S \frac{\partial^{2}}{\partial S \partial V}+\sqrt{2} \rho_{23} v \sigma_{c} \frac{\partial^{2}}{\partial y \partial V}-\sqrt{2} v \Lambda(V) \frac{\partial}{\partial V} \\
\mathcal{L}_{2}= & \frac{\partial}{\partial t}+\frac{1}{2} f(V)^{2} S^{2} \frac{\partial^{2}}{\partial S^{2}}+\frac{1}{2} \sigma_{c}^{2} \frac{\partial^{2}}{\partial y^{2}}+\rho_{12} f(V) S \sigma_{c} \frac{\partial^{2}}{\partial S \partial y}+(r-y) S \frac{\partial}{\partial S} \\
+ & \left(\kappa_{1}(\alpha-y)-\lambda\right) \frac{\partial}{\partial y}-r \cdot=\mathcal{L}_{T F}(f(V)) .
\end{aligned}
$$

Note that $\mathcal{L}_{2}$ is the Schwartz (1997) two factor model operator at the volatility level $f(V)$. We denote this as $\mathcal{L}_{T F}(f(V))$.

With this notation, the partial differential equation (4) for the price of the contingent claim becomes

$$
\left(\frac{1}{\varepsilon} \mathcal{L}_{0}+\frac{1}{\sqrt{\varepsilon}} \mathcal{L}_{1}+\mathcal{L}_{2}\right) P=0
$$

\section{The Formal Expansion}

We expand the solution $P$ of $(9)$ in powers of $\sqrt{\varepsilon}$,

$$
P=P_{0}+\sqrt{\varepsilon} P_{1}+\varepsilon P_{2}+\varepsilon \sqrt{\varepsilon} P_{3}+\cdots .
$$

Our primary interest is in the the first two terms, $P_{0}+\sqrt{\varepsilon} P_{1}$. The terminal condition for the first term is $P_{0}(T, S, y, V)=h(S)$ while the second term has to satisfy $P_{1}(T, S, y, V)=0$.

Substituting equation (10) into equation (9), we obtain

$$
\begin{aligned}
\frac{1}{\varepsilon} \mathcal{L}_{0} P_{0} & +\frac{1}{\sqrt{\varepsilon}}\left(\mathcal{L}_{0} P_{1}+\mathcal{L}_{1} P_{0}\right) \\
& +\left(\mathcal{L}_{0} P_{2}+\mathcal{L}_{1} P_{1}+\mathcal{L}_{2} P_{0}\right) \\
& +\sqrt{\varepsilon}\left(\mathcal{L}_{0} P_{3}+\mathcal{L}_{1} P_{2}+\mathcal{L}_{2} P_{1}\right) \\
& +\cdots \\
& =0
\end{aligned}
$$

\subsection{The Diverging Terms}

To eliminate the terms of order $1 / \varepsilon$, we must have

$$
\mathcal{L}_{0} P_{0}=0 .
$$


Note that the operator $\mathcal{L}_{0}$ takes derivatives with respect to $V$ and that the equation above can only hold if $P_{0}$ is constant with respect to $V$, that is

$$
P_{0}=P_{0}(t, S, y)
$$

and independent of $V$. Similarly, in order to eliminate the terms of order $1 / \sqrt{\varepsilon}$, we must have

$$
\mathcal{L}_{0} P_{1}+\mathcal{L}_{1} P_{0}=0 .
$$

As the operator $\mathcal{L}_{1}$ factors through $\frac{\partial}{\partial V}$ we have $\mathcal{L}_{1} P_{0}=0$. Consequently, we obtain $\mathcal{L}_{0} P_{1}=0$.

Again, because $\mathcal{L}_{0}$ only acts on the $V$ variable, we get

$$
P_{1}=P_{1}(t, S, y),
$$

which also implies that $\mathcal{L}_{1} P_{1}=0$. Therefore, to eliminate the terms of order 1 we must have

$$
\mathcal{L}_{0} P_{2}+\mathcal{L}_{2} P_{0}=0
$$

\subsection{The Zero-Order Term}

Equation (12) is a Poisson equation and only has a solution if

$$
\left\langle\mathcal{L}_{2} P_{0}\right\rangle=0^{3},
$$

where the brackets denote the expectation with respect to the invariant distribution $\chi$ of $V,{ }^{4}$ i.e.

$$
\langle g\rangle=\int_{-\infty}^{\infty} g(z) d \chi(z)
$$

Since $P_{0}$ does not depend on $V$, this means that $\left\langle\mathcal{L}_{2}\right\rangle P_{0}=0$. From the definition of $\mathcal{L}_{2}$ in (8), we can derive that $\left\langle\mathcal{L}_{2}\right\rangle=\mathcal{L}_{T F}(\bar{\sigma})$, where the volatility $\bar{\sigma}$ is defined by

$$
\bar{\sigma}^{2}=\left\langle f^{2}\right\rangle
$$

where $f$ is as in (1).

Therefore, the zero-order term $P_{0}(t, S, y)$ is the solution of the Schwartz (1997) two factor model, identified by

$$
\mathcal{L}_{T F}(\bar{\sigma}) P_{0}=0
$$

\footnotetext{
${ }^{3}$ See discussion in Fouque et al. (2000), page 91.

${ }^{4}$ For operators the expectation involves the coefficient functions.
} 
with the terminal condition $P_{0}(T, S, y)=h(S)$

As the centering condition is satisfied, we have

$$
\mathcal{L}_{2} P_{0}=\mathcal{L}_{2} P_{0}-\left\langle\mathcal{L}_{2} P_{0}\right\rangle=\frac{1}{2}\left(f(V)^{2}-\bar{\sigma}^{2}\right) S^{2} \frac{\partial^{2} P_{0}}{\partial S^{2}}+\rho_{12}(f(V)-\bar{\sigma}) S \sigma_{c} \frac{\partial^{2} P_{0}}{\partial S \partial y} .
$$

Then the second-order correction $P_{2}$ can be identified from (12) as

$$
\begin{aligned}
P_{2}(t, S, y, V)= & -\mathcal{L}_{0}^{-1}\left(\frac{1}{2}\left(f(V)^{2}-\bar{\sigma}^{2}\right) S^{2} \frac{\partial^{2} P_{0}}{\partial S^{2}}+\rho_{12}(f(V)-\bar{\sigma}) S \sigma_{c} \frac{\partial^{2} P_{0}}{\partial S \partial y}\right) \\
= & -\left(\frac{1}{2}\left(\phi_{1}(V)+c_{1}(t, S, y)\right) S^{2} \frac{\partial^{2} P_{0}}{\partial S^{2}}\right. \\
& \left.+\rho_{12}\left(\phi_{2}(V)+c_{2}(t, S, y)\right) S \sigma_{c} \frac{\partial^{2} P_{0}}{\partial S \partial y}\right),
\end{aligned}
$$

where $\phi_{1,2}(V)$ are solutions of the Poisson equations

$$
\begin{gathered}
\mathcal{L}_{0} \phi_{1}=f(V)^{2}-\left\langle f^{2}\right\rangle \\
\mathcal{L}_{0} \phi_{2}=f(V)-\langle f\rangle
\end{gathered}
$$

and $c_{i}(t, S, y), i=1,2$ do not depend on the $V$ variable, but may depend on $(t, S, y)$. This is similar as in Fouque et al. (2000).

\subsection{The First Correction}

Similarly, in order to eliminate the terms of order $\sqrt{\varepsilon}$, we have

$$
\mathcal{L}_{0} P_{3}+\mathcal{L}_{1} P_{2}+\mathcal{L}_{2} P_{1}=0 .
$$

This again is a Poisson equation for $P_{3}$ with respect to $\mathcal{L}_{0}$, which requires the centering condition

$$
\left\langle\mathcal{L}_{1} P_{2}+\mathcal{L}_{2} P_{1}\right\rangle=0 .
$$

The solution for $P_{2}$ is given by equation (15), $P_{1}$ is independent of $V$ and $\left\langle\mathcal{L}_{2}\right\rangle=\mathcal{L}_{T F}(\bar{\sigma})$. We can derive that

$$
\begin{aligned}
\mathcal{L}_{T F}(\bar{\sigma}) P_{1} & =-\left\langle\mathcal{L}_{1} P_{2}\right\rangle \\
& =\frac{1}{2}\left\langle\mathcal{L}_{1} \phi_{1}(V)\right\rangle S^{2} \frac{\partial^{2} P_{0}}{\partial S^{2}}+\rho_{12}\left\langle\mathcal{L}_{1} \phi_{2}(V)\right\rangle S \sigma_{c} \frac{\partial^{2} P_{0}}{\partial S \partial y} .
\end{aligned}
$$

Note again that $\mathcal{L}_{1}$ factors through $\frac{\partial}{\partial V}$ and $c_{i}(t, S, y)$ does not depend on $V$, hence we can derive that $\mathcal{L}_{1} c_{i}=0$. 
Then the problem is to determine the expressions $\left\langle\mathcal{L}_{1} \phi_{1}(V)\right\rangle$ and $\left\langle\mathcal{L}_{1} \phi_{2}(V)\right\rangle$,

$$
\begin{aligned}
\left\langle\mathcal{L}_{1} \phi_{i}(V) \cdot\right\rangle & =\sqrt{2} \rho_{13} v\left\langle f(V) \phi_{i}^{\prime}(V)\right\rangle S \frac{\partial}{\partial S} \\
& +\sqrt{2} \rho_{23} v \sigma_{c}\left\langle\phi_{i}^{\prime}(V)\right\rangle \frac{\partial}{\partial y}-\sqrt{2} v\left\langle\Lambda(V) \phi_{i}^{\prime}(V)\right\rangle . \quad i=1,2
\end{aligned}
$$

Finally, we can deduce that

$$
\begin{aligned}
\mathcal{L}_{T F}(\bar{\sigma}) P_{1} & =\frac{\sqrt{2}}{2} \rho_{13} v\left\langle f \phi_{1}^{\prime}\right\rangle S^{3} \frac{\partial^{3} P_{0}}{\partial S^{3}} \\
& +\left(\frac{\sqrt{2}}{2} \rho_{23} v\left\langle\phi_{1}^{\prime}\right\rangle+\sqrt{2} \rho_{12} \rho_{13} v\left\langle f \phi_{2}^{\prime}\right\rangle\right) S^{2} \sigma_{c} \frac{\partial^{3} P_{0}}{\partial S^{2} \partial y} \\
& +\sqrt{2} \rho_{12} \rho_{23} v\left\langle\phi_{2}^{\prime}\right\rangle S \sigma_{c}^{2} \frac{\partial^{3} P_{0}}{\partial S \partial y^{2}} \\
& +\left(\sqrt{2} \rho_{13} v\left\langle f \phi_{1}^{\prime}\right\rangle-\frac{\sqrt{2}}{2} v\left\langle\Lambda \phi_{1}^{\prime}\right\rangle\right) S^{2} \frac{\partial^{2} P_{0}}{\partial S^{2}} \\
& +\left(\sqrt{2} \rho_{12} \rho_{13} v\left\langle f \phi_{2}^{\prime}\right\rangle-\sqrt{2} \rho_{12} v\left\langle\Lambda \phi_{2}^{\prime}\right\rangle\right) S \sigma_{c} \frac{\partial^{2} P_{0}}{\partial S \partial y} .
\end{aligned}
$$

Now we introduce the first correction term, $\hat{P}_{1}(t, S, y)=\sqrt{\varepsilon} P_{1}(t, S, y)$, and write equation (22) as

$$
\mathcal{L}_{T F}(\bar{\sigma}) \hat{P}_{1}=G(t, S, y)
$$

where $G(t, S, y)$ reflects upon the right hand side of (22). The solution for $\hat{P}_{1}(t, S, y)$ is $-(T-t) G$ with boundary condition. This can be easily verified from the identity,

$$
\mathcal{L}_{T F}(\bar{\sigma})(-(T-t) G)=G-(T-t) \mathcal{L}_{T F}(\bar{\sigma}) G .
$$

The second term on the right side of equation (24) is equal to zero (see appendix A for detail).

Therefore, the solution for the second term $\sqrt{\varepsilon} P_{1}$ in the expansion equation (10) is

$$
\begin{aligned}
\sqrt{\varepsilon} P_{1}= & -(T-t)\left(A_{2} S^{2} \frac{\partial^{2} P_{0}}{\partial S^{2}}+A_{3} S \frac{\partial^{2} P_{0}}{\partial S \partial y}+A_{4} S^{3} \frac{\partial^{3} P_{0}}{\partial S^{3}}\right. \\
& \left.+A_{5} S^{2} \frac{\partial^{3} P_{0}}{\partial S^{2} \partial y}+A_{6} S \frac{\partial^{3} P_{0}}{\partial S \partial y^{2}}\right)
\end{aligned}
$$


where $A_{2}, A_{3}, A_{4}, A_{5}$ and $A_{6}$ are five coefficients, given in terms of $\kappa_{2}=1 / \varepsilon$ by

$$
\begin{gathered}
A_{2}=\frac{v}{\sqrt{2 \kappa_{2}}}\left(2 \rho_{13}\left\langle f \phi_{1}^{\prime}\right\rangle-\left\langle\Lambda \phi_{1}^{\prime}\right\rangle\right) \\
A_{3}=\left(\frac{\sqrt{2} \rho_{12} \rho_{13}}{\sqrt{\kappa_{2}}}\left\langle f \phi_{2}^{\prime}\right\rangle-\frac{\sqrt{2} \rho_{12}}{\sqrt{\kappa_{2}}\left\langle\Lambda \phi_{2}^{\prime}\right\rangle}\right) \sigma_{c} v \\
A_{4}=\frac{\rho_{13} v}{\sqrt{2 \kappa_{2}}}\left\langle f \phi_{1}^{\prime}\right\rangle \\
A_{5}=\left(\frac{\rho_{23}}{\sqrt{2 \kappa_{2}}}\left\langle\phi_{1}^{\prime}\right\rangle+\frac{\sqrt{2} \rho_{12} \rho_{13}}{\sqrt{\kappa_{2}}}\left\langle f \phi_{2}^{\prime}\right\rangle\right) \sigma_{c} v \\
A_{6}=\frac{\sqrt{2} \rho_{12} \rho_{23}}{\sqrt{\kappa_{2}}}\left\langle\phi_{2}^{\prime}\right\rangle \sigma_{c}^{2} v .
\end{gathered}
$$

Therefore, the corrected price is given explicitly by

$$
\begin{aligned}
P=P_{0}-(T-t)\left(A_{2} S^{2} \frac{\partial^{2} P_{0}}{\partial S^{2}}\right. & +A_{3} S \frac{\partial^{2} P_{0}}{\partial S \partial y}+A_{4} S^{3} \frac{\partial^{3} P_{0}}{\partial S^{3}} \\
& \left.+A_{5} S^{2} \frac{\partial^{3} P_{0}}{\partial S^{2} \partial y}+A_{6} S \frac{\partial^{3} P_{0}}{\partial S \partial y^{2}}\right)
\end{aligned}
$$

where $P_{0}$ is the Schwartz (1997) two factor model price with constant volatility $\bar{\sigma}$.

The function $\phi_{1}^{\prime}$ can be computed analogous as in Fouque et al. (2000) from equation (16),

$$
\phi_{1}^{\prime}(V)=\frac{1}{v^{2} \Phi(V)} \int_{-\infty}^{.}\left(f^{2}-\left\langle f^{2}\right\rangle\right) \Phi,
$$

where $\Phi(V)$ is the probability density of the $\mathcal{N}\left(m, v^{2}\right)$-invariant distribution.

Note that we have zero on both sides when the interval of integration is infinite.

Therefore, with a particular choice of a function $f$ (e.g., $f(V)=V$ ) and boundary condition, we can find,

$$
\begin{aligned}
\left\langle f \phi_{1}^{\prime}\right\rangle & =\left\langle\frac{f}{v^{2} \Phi} \int_{-\infty}^{\cdot}\left(f^{2}-\left\langle f^{2}\right\rangle\right) \Phi\right\rangle \\
& =\frac{1}{v^{2}} \int_{-\infty}^{+\infty} f \int_{-\infty}\left(f^{2}-\left\langle f^{2}\right\rangle\right) \Phi \\
& =-\frac{1}{v^{2}}\left\langle X\left(f^{2}-\left\langle f^{2}\right\rangle\right)\right\rangle,
\end{aligned}
$$


where $X$ is the indefinite integral of $f$ (i.e. $X^{\prime}=f$ ), and

$$
\left\langle\phi_{1}^{\prime}\right\rangle=-\frac{1}{v^{2}}\left\langle V\left(f^{2}-\left\langle f^{2}\right\rangle\right)\right\rangle
$$

Similarly, the solution for $\phi_{2}^{\prime}$ is,

$$
\phi_{2}^{\prime}=\frac{1}{v^{2} \Phi} \int_{-\infty}^{\cdot}(f-\langle f\rangle) \Phi .
$$

Therefore, we can find the solution for $\left\langle f \phi_{2}^{\prime}\right\rangle$,

$$
\left\langle f \phi_{2}^{\prime}\right\rangle=-\frac{1}{v^{2}}\langle X(f-\langle f\rangle)\rangle
$$

and

$$
\left\langle\phi_{2}^{\prime}\right\rangle=-\frac{1}{v^{2}}\langle V(f-\langle f\rangle)\rangle
$$

Assuming the market price of volatility risk $\gamma$ is 0 and $f(V)=V$, the quantities $A_{2}, A_{3}, A_{4}, A_{5}$ and $A_{6}$ can be obtained analytically by the remaining model parameters (see appendix $\mathrm{B}$ for detail):

$$
\begin{gathered}
A_{2}=-\frac{2 \rho_{13} v}{\sqrt{2 \kappa_{2}}}\left(2 m^{2}+v^{2}\right) \\
A_{3}=-\frac{\sqrt{2} \rho_{12} \rho_{13} \sigma_{c} v m}{\sqrt{\kappa_{2}}} \\
A_{4}=-\frac{\rho_{13} v}{\sqrt{2 \kappa_{2}}}\left(2 m^{2}+v^{2}\right) \\
A_{5}=-\sigma_{c} v\left(\frac{2 m \rho_{23}}{\sqrt{2 \kappa_{2}}}+\frac{\sqrt{2} \rho_{12} \rho_{13} m}{\sqrt{\kappa_{2}}}\right) \\
A_{6}=-\frac{\sqrt{2} \rho_{12} \rho_{23} \sigma_{c}^{2} v}{\sqrt{\kappa_{2}}} .
\end{gathered}
$$




\section{European Commodity Call Options}

Formulas for pricing European options on commodity futures in the Schwartz (1997) two-factor model (without stochastic volatility) have been presented in Miltersen and Schwartz (1998) and Hilliard and Reis (1998).

Here we assume that the European call option $C$ is considered at time zero with maturity $t$, exercise price $K$ and written on a commodity futures contract with maturity $T$. Using $\bar{\sigma}$ to substitute for the constant spot price volatility, the value of a European call option on a futures in the Schwartz (1997) two-factor model is given by

$$
P_{0}(0, t, T)=e^{-r t}\left[F(0, T) N\left(d_{1}\right)-K N\left(d_{2}\right)\right]
$$

with

$$
\begin{gathered}
d_{1}=\frac{\ln (F(0, T) / K)+\frac{1}{2} \sigma^{2}}{\sigma}, \quad d_{2}=d_{1}-\sigma \\
\sigma^{2}(0, t, T)=\bar{\sigma}^{2} t \\
\quad-\frac{2 \rho_{12} \bar{\sigma} \sigma_{c}}{\kappa_{1}}\left[t-\frac{\left(e^{-\kappa_{1}(T-t)}-e^{-k_{1} T}\right)}{\kappa_{1}}\right] \\
+\frac{\sigma_{c}^{2}}{\kappa_{1}^{2}}\left[t-\frac{2}{\kappa_{1}}\left(e^{-\kappa_{1}(T-t)}-e^{-k_{1} T}\right)\right. \\
\left.+\frac{1}{2 \kappa_{1}}\left(e^{-2 \kappa_{1}(T-t)}-e^{-2 \kappa_{1} T}\right)\right]
\end{gathered}
$$

and $N(\cdot)$ being the cumulative standard Normal distribution function.

Hilliard and Reis (1998) also presented a simple expression for the price of a futures contracts under the assumption of constant spot volatility. In our case, the value of a futures contract at time zero is given by

$$
F\left(S_{0}, y_{0}, 0, T\right)=S_{0} e^{A(T)+H(T) y_{0}}
$$

with

$$
\begin{aligned}
A(T)= & \left(r-\tilde{\alpha}+\frac{\sigma_{c}^{2}}{2 \kappa_{1}^{2}}-\frac{\bar{\sigma} \sigma_{c} \rho_{12}}{\kappa_{1}}\right) T+\frac{\sigma_{c}^{2}\left(1-e^{-2 \kappa_{1} T}\right)}{4 \kappa_{1}^{3}} \\
& +\left(\kappa_{1} \tilde{\alpha}+\bar{\sigma} \sigma_{c} \rho_{12}-\frac{\sigma_{c}^{2}}{\kappa_{1}}\right) \frac{1-e^{-\kappa_{1} T}}{\kappa_{1}^{2}}, \\
H(T)= & -\frac{1-e^{-\kappa_{1} T}}{\kappa_{1}},
\end{aligned}
$$


where $\tilde{\alpha}=\alpha-\lambda / \kappa_{1}$.

\section{Asymptotic Solution in Terms of Futures Prices}

In this section we rewrite equation (31) in terms of futures rather than spot prices. Technically, this amounts to changing the set of state variables in the model from $(S, y, V)$ to $(F, y, V)$, where $F$ denotes the futures price for a given maturity. The advantage of this is that the asymptotic formula then admits a feature of symmetry, which is useful in calculations.

As $P_{0}$ is given in form of an explicit analytic expression, we can compute the derivatives $\frac{\partial^{2} P_{0}}{\partial S^{2}}, \frac{\partial^{2} P_{0}}{\partial S \partial y}, \frac{\partial^{3} P_{0}}{\partial S^{3}}, \frac{\partial^{3} P_{0}}{\partial S^{2} \partial y}$ and $\frac{\partial^{3} P_{0}}{\partial S \partial y^{2}}$ in equation (31). We may then compute the Delta of the $P_{0}$ term as follows:

$$
\begin{aligned}
\frac{\partial P_{0}}{\partial S} & =\frac{\partial P_{0}}{\partial F} \cdot \frac{\partial F}{\partial S} \\
& =e^{-r t} N\left(d_{1}\right)\left(e^{A+H y}\right) \\
& =\frac{\partial P_{0}}{\partial F} \cdot \frac{F}{S} .
\end{aligned}
$$

Similar, we obtain for the Gamma,

$$
\begin{aligned}
\frac{\partial^{2} P_{0}}{\partial S^{2}} & =\frac{\partial^{2} P_{0}}{\partial F^{2}} \cdot\left(\frac{\partial F}{\partial S}\right)^{2}+\frac{\partial P_{0}}{\partial F} \cdot \frac{\partial^{2} F}{\partial S^{2}} \\
& =e^{-r t} \frac{N^{\prime}\left(d_{1}\right)}{F \sigma}\left(e^{A+H y}\right)^{2} \\
& =e^{-r t} \frac{N^{\prime}\left(d_{1}\right)}{S \sigma}\left(e^{A+H y}\right) \\
& =\frac{\partial^{2} P_{0}}{\partial F^{2}} \cdot\left(\frac{F}{S}\right)^{2},
\end{aligned}
$$

where we note that $\frac{\partial^{2} F}{\partial S^{2}}=0$. 
Further, for the Speed,

$$
\begin{aligned}
\frac{\partial^{3} P_{0}}{\partial S^{3}} & =\frac{\partial^{3} P_{0}}{\partial F^{3}} \cdot\left(\frac{\partial F}{\partial S}\right)^{3}+3 \frac{\partial^{2} P_{0}}{\partial F^{2}} \cdot \frac{\partial F}{\partial S} \cdot \frac{\partial^{2} F}{\partial S^{2}}+\frac{\partial P_{0}}{\partial F} \frac{\partial^{3} F}{\partial S^{3}} \\
& =-e^{-r t} \frac{N^{\prime}\left(d_{1}\right)}{F^{2} \sigma}\left(\frac{d_{1}}{\sigma}+1\right)\left(e^{A+H y}\right)^{3} \\
& =-e^{-r t} \frac{N^{\prime}\left(d_{1}\right)}{S^{2} \sigma}\left(\frac{d_{1}}{\sigma}+1\right)\left(e^{A+H y}\right) \\
& =\frac{\partial^{3} P_{0}}{\partial F^{3}} \cdot\left(\frac{F}{S}\right)^{3}
\end{aligned}
$$

noting that $\frac{\partial^{3} F}{\partial S^{3}}=0$. More generally, it is possible to show that

$$
\frac{\partial^{n} P_{0}}{\partial S^{n}}=\frac{\partial^{n} P_{0}}{\partial F^{n}}\left(\frac{F}{S}\right)^{n}
$$

We can also compute the cross derivatives

$$
\frac{\partial^{2} P_{0}}{\partial S \partial y}=\frac{\partial P_{0}}{\partial F} \frac{F H}{S}+\frac{\partial^{2} P_{0}}{\partial F^{2}} \frac{F^{2} H}{S}
$$

and

$$
\frac{\partial^{3} P_{0}}{\partial S^{2} \partial y}=\frac{\partial^{2} P_{0}}{\partial F^{2}}\left(1-\frac{d_{1}}{\sigma}\right) H\left(\frac{F}{S}\right)^{2} \frac{\partial^{2} P_{0}}{\partial F^{2}}
$$

as well as

$$
\frac{\partial^{3} P_{0}}{\partial S \partial y^{2}}=\frac{\partial P_{0}}{\partial F} \frac{F H}{S}+\frac{\partial^{2} P_{0}}{\partial F^{2}}\left(\frac{F^{2} H}{S}+\frac{F^{2} H^{2}}{S \sigma}\left(1-d_{1}\right)\right) .
$$

Therefore, the asymptotic expansion including the correction term, (31), can be expressed as in terms of futures prices as

$$
\begin{aligned}
P & =P_{0}-(T-t)\left(A_{2} F^{2} \frac{\partial^{2} P_{0}}{F^{2}}+A_{3}\left(F H \frac{\partial P_{0}}{\partial F}+F^{2} H \frac{\partial^{2} P_{0}}{\partial F^{2}}\right)\right. \\
& +A_{4} F^{3} \frac{\partial^{3} P_{0}}{\partial F^{3}}+A_{5} F^{2} H\left(1-\frac{d_{1}}{\sigma}\right) \frac{\partial^{2} P_{0}}{\partial F^{2}} \\
& \left.+A_{6}\left(F H \frac{\partial P_{0}}{\partial F}+F^{2} H \frac{\partial^{2} P_{0}}{\partial F^{2}}+\frac{F^{2} H^{2}}{\sigma}\left(1-d_{1}\right) \frac{\partial^{2} P_{0}}{\partial F^{2}}\right)\right)
\end{aligned}
$$

where $P_{0}$ is the Schwartz (1997) two factor model price with constant volatility $\bar{\sigma}$ 


\section{Asymptotic Results on Simulated Data}

In order to demonstrate the accuracy of our formula, we present some numerical examples in this section, taking account of different option positions. We compare the results obtained from our asymptotic formula to those obtained from the Schwartz (1997) two factor model with constant volatility (explicit formula) and those from Monte Carlo simulation for the full model with stochastic volatility.

In the process of the Monte Carlo simulation we employed the Euler Maruyama discretization for the spot, convenience yield and stochastic volatility dynamic according to

$$
\begin{aligned}
& V_{t}=V_{t-\Delta t}+\left(\kappa_{2}\left(m-V_{t-\Delta t}\right)\right) \Delta t+\beta d W_{3_{t-\Delta t}} \\
& y_{t}=y_{t-\Delta t}+\left(\kappa_{1}\left(\alpha-y_{t-\Delta t}\right)-\lambda\right) \Delta t+\sigma_{c} d W_{2_{t-\Delta t}} \\
& S_{t}=S_{t-\Delta t}\left(1+\left(r-y_{t-\Delta t}\right) \Delta t+V_{t-\Delta t} d W_{1_{t-\Delta t}}\right)
\end{aligned}
$$

and generating a given correlation structure through Cholesky decomposition

$$
\left[\begin{array}{ccc}
1 & 0 & 0 \\
\rho_{12} & \sqrt{1-\rho_{12}^{2}} & 0 \\
\rho_{13} & \frac{\rho_{23}-\rho_{12} \rho_{13}}{\sqrt{1-\rho_{12}^{2}}} & \sqrt{1-\rho_{13}^{2}-\left(\frac{\rho_{23}-\rho_{12} \rho_{13}}{\sqrt{1-\rho_{12}^{2}}}\right)^{2}}
\end{array}\right]\left[\begin{array}{c}
d \bar{W}_{1} \\
d \bar{W}_{2} \\
d \bar{W}_{3}
\end{array}\right],
$$

with $\bar{W}_{i}, i=1,2,3$ independent Brownian motions.

We assume that the initial spot price is 100 , the initial convenience yield is 0.05 , the initial spot volatility is 0.8944 and both futures contracts' and futures options contracts' maturity is 1 year. All parameters of the model are listed in table (1).

\begin{tabular}{cccc} 
Table 1: Parameter Choices for Three Factor Mode \\
\hline \hline Parameter & Value & Parameter & Value \\
\hline$r$ & 0.02 & $\kappa_{1}$ & 0.8 \\
$\tilde{\alpha}$ & 0.3 & $\sigma_{c}$ & 1.1 \\
$\kappa_{2}$ & 5 & $m$ & 0.05 \\
$\beta$ & 0.5 & $\rho_{12}$ & 0.3 \\
$\rho_{13}$ & -0.7 & $\rho_{23}$ & -0.5 \\
\hline
\end{tabular}

We can then estimate the average spot volatility $\bar{\sigma}$ from Monte Carlo simulation as 0.2162 and compute the long term standard deviation of volatility $v$ as 0.1581 . From equations (38) to (42), we can find the following values 
for the $A_{i}$ 's : $A_{2}=0.0021, A_{3}=0.0012, A_{4}=0.001, A_{5}=0.0039$ and $A_{6}=0.0182$.

With these parameters, futures call option prices have been computed by the asymptotic method (Asymptotic) and compared to Monte Carlo prices (MC) and the prices computed from the Schwartz (1997) two factor model (TF) without stochastic volatility. We choose the Monte Carlo simulation results as our benchmark price. The variable gap1 represents the error $(\%)$ between the MC and Asymptotic prices while gap2 represents the error(\%) between the prices for MC and TF.

Table 2 shows that the results of the asymptotic method are in general better than those of the standard Schwartz (1997) two factor model, except for the deep in the money options with strike prices of 80 . More specifically, the error for asymptotic results seems to vary in a non-monotonic way through moneyness of the option, taking a minimum for 'at the money' options, while the error for the Schwartz (1997) two factor model increases monotonically as the strike price increases, from $1.86 \%$ to $8.9 \%$. For at the money options, the result obtained from the asymptotic method is roughly 4 times better than the result obtained from the Schwartz (1997) two factor model. This is particularly important as these options are generally the most liquid options on the market, having attached a large weight in the calibration of the model.

Table 2: Futures options prices

This table shows the numerical results obtained from Monte Carlo, asymptotic formula and Schwartz (1997) two factor formula for options with 1 year maturity. The initial spot price is 100 , and strike price is 80 , 90, 100, 110 and 120 accordingly. The variable gap1 represents the error between Monte Carlo results (MC) and asymptotic results (Asymptotic), gap2 represents the error between Monte Carlo (MC) and standard

Schwartz (1997) two factor results (TF)

\begin{tabular}{lccccc}
\hline \hline $\mathrm{S}=100$ & $\mathrm{MC}$ & Asymptotic & gap1 & TF & gap2 \\
\hline $\mathrm{K}=80$ & 27.0215 & 27.8974 & $3.24 \%$ & 26.5188 & $1.86 \%$ \\
$\mathrm{~K}=90$ & 22.1095 & 22.4095 & $1.36 \%$ & 21.3547 & $3.41 \%$ \\
$\mathrm{~K}=100$ & 18.0338 & 17.8067 & $1.26 \%$ & 17.1092 & $5.13 \%$ \\
$\mathrm{~K}=110$ & 14.684 & 14.0133 & $4.57 \%$ & 13.6617 & $6.96 \%$ \\
$\mathrm{~K}=120$ & 11.95 & 10.9333 & $8.51 \%$ & 10.8869 & $8.90 \%$ \\
\hline
\end{tabular}




\section{Asymptotic Results on Market Data}

\subsection{Data}

We consider European natural gas futures option as traded on the Chicago Mercantile Exchange (CME) in order to test our asymptotic formula and to test specifically whether the asymptotic formula provides any improvement in terms of overall fit to market data, as compared to the Schwartz (1997) two-factor formula. The chosen data cover the period from 27/03/2012 to 26/09/2013, with 2 year, 1 year, 0.75 year and 0.5 maturities. Table 3 below shows futures prices of a particular futures contract maturing in March 2014 at dates $27 / 12 / 2012,26 / 03 / 2013,26 / 06 / 2013$ and 26/09/2013 respectively. The interest rate $r$ during this time period is set at $0.5 \%^{5}$.

\section{Table 3: Gas Futures Prices}

This table presents the natural gas futures prices on $27 / 03 / 2012(\mathrm{~T}=2)$, $26 / 03 / 2013(\mathrm{~T}=1), 26 / 06 / 2013(\mathrm{~T}=0.75)$ and $26 / 09 / 2013(\mathrm{~T}=0.5)$.

\begin{tabular}{cc}
\hline \hline & $\begin{array}{c}\text { Gas Future } \\
\text { Prices }\end{array}$ \\
\hline $\mathrm{T}=2$ & 3.82 \\
$\mathrm{~T}=1$ & 4.101 \\
$\mathrm{~T}=0.75$ & 3.934 \\
$\mathrm{~T}=0.5$ & 3.793 \\
\hline
\end{tabular}

Since the natural gas futures prices are centered around 4, we chose natural gas futures options contracts with strike prices $2.5,3,3.5,4,4.5$ and 5 to study the performance of the asymptotic pricing formula for different levels of moneyness and overall. Table 4 displays the market prices of the natural gas futures options corresponding to the futures prices in Table 3 and the chosen strike prices.

\footnotetext{
${ }^{5}$ The US Dollar LIBOR average rates in 2013 for 6 month, 9 month and 12 month maturities were $0.409 \%, 0.601 \%$ and $0.683 \%$ respectively, $0.5 \%$ is therefore a reasonable value for short to medium term interest rates during this period. Our model features a constant interest rate $r$ and therefore does not permit too much flexibility to be fitted to arbitrary term structures. However fitting arbitrary term structures is also not the intention of our model. Our aim is to obtain a good general fit of option prices for short to medium term options. Nevertheless, the inclusion of stochastic interest rates into our methodology presents an interesting avenue for further research.
} 


\subsection{Calibration}

Both models, standard Schwartz (1997) two factor model and our asymptotic two factor model with stochastic volatility, have been calibrated to the market prices using non-linear least squares. Parameters for both models have been estimated and are presented in Table 5 .

As Fouque et al. (2000) show, the accuracy of the approximation depends on the ergodicity of the process $V_{t}$, i.e. $\lim _{t \rightarrow \infty} \frac{1}{t} \int_{0}^{t} g\left(V_{s}\right) d s=\langle g\rangle$. In how far $\frac{1}{t} \int_{0}^{t} g\left(V_{s}\right) d s$ approximates $\langle g\rangle$ therefore depends on the mean reversion speed $\kappa_{2}$ in addition to the time to maturity. In this case, the estimate of $\kappa_{2}$ and the maturities considered provide a good fit. The residual sum of squares (RSS) which measures the overall fit of the calibration for the chosen natural gas

Table 4: Gas Futures Call Option Prices

This table presents the prices of natural gas futures options (GKJ4) on $27 / 03 / 2012(\mathrm{~T}=2), 26 / 03 / 2013(\mathrm{~T}=1), 26 / 06 / 2013(\mathrm{~T}=0.75)$ and $26 / 09 / 2013(\mathrm{~T}=0.5)$, with strike price $K$ set at $2.5,3,3.5,4,4.5,5$.

\begin{tabular}{ccccccc}
\hline \hline & $\mathrm{K}=2.5$ & $\mathrm{~K}=3$ & $\mathrm{~K}=3.5$ & $\mathrm{~K}=4$ & $\mathrm{~K}=4.5$ & $\mathrm{~K}=5$ \\
\hline $\mathrm{T}=2$ & 1.3874 & 0.9976 & 0.6871 & 0.4623 & 0.3106 & 0.2068 \\
$\mathrm{~T}=1$ & 1.6226 & 1.1648 & 0.7767 & 0.4821 & 0.2816 & 0.1583 \\
$\mathrm{~T}=0.75$ & 1.4377 & 0.9719 & 0.5798 & 0.3086 & 0.1534 & 0.0745 \\
$\mathrm{~T}=0.5$ & 1.2978 & 0.8325 & 0.45 & 0.2065 & 0.0837 & 0.0314 \\
\hline
\end{tabular}

Table 5: Parameter Estimation from Market Data for Asymptotic Two Factor Model and Standard Two Factor Model

\begin{tabular}{ccc}
\hline \hline Parameter & $\begin{array}{c}\text { Asymptotic } \\
\text { formula }\end{array}$ & $\begin{array}{c}\text { Two factor } \\
\text { model }\end{array}$ \\
\hline $\bar{\sigma}$ & 0.461 & 0.498 \\
$\sigma_{c}$ & 3.1124 & 3.5793 \\
$\kappa_{1}$ & 6.7193 & 6.4295 \\
$\kappa_{2}$ & 2.3604 & $\mathrm{~N} / \mathrm{A}$ \\
$\rho_{12}$ & 0.8817 & 0.8919 \\
$\rho_{13}$ & -0.8912 & $\mathrm{~N} / \mathrm{A}$ \\
$\rho_{23}$ & -0.1827 & $\mathrm{~N} / \mathrm{A}$ \\
$m$ & 0.2638 & $\mathrm{~N} / \mathrm{A}$ \\
$v$ & 0.0188 & $\mathrm{~N} / \mathrm{A}$ \\
\hline
\end{tabular}


futures call options for the asymptotic solution and the standard Schwartz (1997) two factor model are 0.0018 and 0.0026 respectively. While the difference appears to be small in absolute terms, it becomes very significant with large trading volume. In relative terms, and this is the important conclusion, the asymptotic method improves overall accuracy by roughly $30 \%$, compared with the standard Schwartz (1997) two factor model. The benefit from using the asymptotic method is therefore evident. Further, looking at different maturities separately, we observe from Table 7 , that the value of RSS for the asymptotic two factor model are lower than the corresponding RSS for the standard Schwartz (1997) two factor model in all four maturities cases. Especially, for long maturities $(T=2)$, the performance of the asymptotic method is significantly better than the performance of the standard Schwartz (1997) two factor model. This improved fit comes at virtually no extra cost in computational effort, as our asymptotic formula is explicit and no more complex that the standard Schwartz (1997) two factor formula.

\section{Conclusion}

In this paper, we derived an asymptotic option pricing formula for a Schwartz (1997) like model with stochastic convenience yield and stochastic volatility. The formula can be expressed both in terms of spot price and futures price. We demonstrate that our formula provides an excellent approximation through use of both simulated and real market data. Compared with corresponding results for the standard Schwartz (1997) two factor model, the asymptotic model solution improves overall accuracy by about $30 \%$. The

\section{Table 6: Natural Gas Futures Call Option Prices from Asymptotic} Two Factor Solution

This table presents the prices of natural gas futures call options from asymptotic two factor model on $27 / 03 / 2012(\mathrm{~T}=2), 26 / 03 / 2013(\mathrm{~T}=1)$, $26 / 06 / 2013(\mathrm{~T}=0.75)$ and $26 / 09 / 2013(\mathrm{~T}=0.5)$, with strike price $(\mathrm{K}), 2.5,3$, $3.5,4,4.5,5$.

\begin{tabular}{ccccccc}
\hline \hline & $\mathrm{K}=2.5$ & $\mathrm{~K}=3$ & $\mathrm{~K}=3.5$ & $\mathrm{~K}=4$ & $\mathrm{~K}=4.5$ & $\mathrm{~K}=5$ \\
\hline $\mathrm{T}=2$ & 1.3885 & 1.0009 & 0.6950 & 0.4691 & 0.3103 & 0.2022 \\
$\mathrm{~T}=1$ & 1.6149 & 1.1526 & 0.7609 & 0.4675 & 0.2706 & 0.1495 \\
$\mathrm{~T}=0.75$ & 1.4441 & 0.9791 & 0.5950 & 0.3261 & 0.1640 & 0.0772 \\
$\mathrm{~T}=0.5$ & 1.2989 & 0.8277 & 0.4477 & 0.2060 & 0.0827 & 0.0298 \\
\hline
\end{tabular}


improvement is even better, by a factor of 4, for options with long maturities. As our asymptotic formula is explicit and analytic, its application does not increase the computing time, compared to that from the standard Schwartz (1997) two factor model. In conclusion we recommend application of our asymptotic formula instead of the standard Schwartz (1997) two factor formula. We further believe that our asymptotic formula will lead to better performance in hedging, but leave this for future research.

\section{Appendix A}

We prove that $\mathcal{L}_{T F}(\bar{\sigma}) G$ in equation (24) is zero.

In equation $(23)$, the operator $\mathcal{L}_{T F}(\bar{\sigma})$ is expressed as in equation (8), acting on the variables $S$ and $y$. In fact, it can be transformed into an operator which only acts on $F$, that is,

$$
\mathcal{L}_{T F}(\bar{\sigma})=\frac{\partial}{\partial t}+\frac{1}{2} \sigma^{2} F^{2} \frac{\partial^{2}}{\partial F^{2}}-r
$$

where $F=S e^{A+H y}, \sigma=\sqrt{\bar{\sigma}^{2}+H^{2} \sigma_{c}^{2}+2 \rho_{12} \bar{\sigma} \sigma_{c} H} \cdot{ }^{6}$

Similarly, $G$ can also be rewritten as a function of $F$, that is,

$$
\begin{aligned}
& \left(A_{2} F^{2} \frac{\partial^{2} P_{0}}{F^{2}}+A_{3}\left(F H \frac{\partial P_{0}}{\partial F}+F^{2} H \frac{\partial^{2} P_{0}}{\partial F^{2}}\right)+A_{4} F^{3} \frac{\partial^{3} P_{0}}{\partial F^{3}}+A_{5} F^{2} H\left(1-\frac{d_{1}}{\sigma}\right) \frac{\partial^{2} P_{0}}{\partial F^{2}}\right. \\
+ & A_{6}\left(F H \frac{\partial P_{0}}{\partial F}+F^{2} H \frac{\partial^{2} P_{0}}{\partial F^{2}}+\frac{F^{2} H^{2}}{\sigma}\left(1-d_{1}\right) \frac{\partial^{2} P_{0}}{\partial F^{2}}\right) .
\end{aligned}
$$

${ }^{6}$ Note that $H$ and hence $\sigma$ in (47) depend on the time to maturity $T-t$. Hence (47) is a Black-Scholes equation with time dependent volatility.

Table 7: RSS for Asymptotic Two Factor Model and Standard Two Factor Model in terms of Time Maturity

\begin{tabular}{ccc}
\hline \hline $\begin{array}{c}\text { Time } \\
\text { maturity }\end{array}$ & $\begin{array}{c}\text { Asymptotic } \\
\text { formula }\end{array}$ & $\begin{array}{c}\text { Two factor } \\
\text { model }\end{array}$ \\
\hline$T=2$ & 0.0001 & 0.0004 \\
$T=1$ & 0.0009 & 0.0011 \\
$T=0.75$ & 0.0007 & 0.0010 \\
$T=0.5$ & 0.0001 & 0.0001 \\
Sum & 0.0018 & 0.0026 \\
\hline
\end{tabular}


We can therefore conclude that the second term $\mathcal{L}_{T F}(\bar{\sigma}) G$ in equation (24) is zero since,

$$
\mathcal{L}_{T F}(\bar{\sigma})\left(F^{n} \frac{\partial^{n} P_{0}}{\partial F^{n}}\right)=F^{n} \frac{\partial^{n}}{\partial F^{n}} \mathcal{L}_{T F}(\bar{\sigma}) P_{0}=0 .
$$

Equation (49) can be proved by following the method provided in Ting (2013).

\section{Appendix B}

In order to compute $A_{2}, A_{3}, A_{4}, A_{5}$ and $A_{6}$ analytically, we must compute the expressions $\left\langle f \phi_{1}^{\prime}\right\rangle,\left\langle\phi_{1}^{\prime}\right\rangle,\left\langle f \phi_{2}^{\prime}\right\rangle$ and $\left\langle\phi_{2}^{\prime}\right\rangle$.

Note that we assume $f(V)=V$ and that $V$ is distributed according to a normal distribution $N\left(m, v^{2}\right)$. Therefore

$$
\begin{aligned}
\left\langle f \phi_{1}^{\prime}\right\rangle & =-\frac{1}{v^{2}}\left\langle F\left(f^{2}-\left\langle f^{2}\right\rangle\right)\right\rangle \\
& =-\frac{1}{v^{2}} \int_{-\infty}^{+\infty} \frac{1}{2} V^{2}\left(V^{2}-\left\langle V^{2}\right\rangle\right) \Phi(V) d V \\
& =-\frac{1}{v^{2}}\left(\frac{1}{2}\left(\mathbb{E}\left(V^{4}\right)-\mathbb{E}\left(V^{2}\right) \mathbb{E}\left(V^{2}\right)\right)\right) \\
& =-\frac{1}{v^{2}}\left(\frac{1}{2}\left(m^{4}+6 m^{2} v^{2}+3 v^{4}-m^{4}-2 m^{2} v^{2}-v^{4}\right)\right) \\
& =-\left(2 m^{2}+v^{2}\right) .
\end{aligned}
$$

Similarly, the expressions for $\left\langle\phi_{1}^{\prime}\right\rangle,\left\langle f \phi_{2}^{\prime}\right\rangle$ and $\left\langle\phi_{2}^{\prime}\right\rangle$ are

$$
\begin{aligned}
& \left\langle\phi_{1}^{\prime}\right\rangle=-2 m \\
& \left\langle f \phi_{2}^{\prime}\right\rangle=-m
\end{aligned}
$$

and,

$$
\left\langle\phi_{2}^{\prime}\right\rangle=-1
$$

The expressions for $A_{2}, A_{3}, A_{4}, A_{5}$ and $A_{6}$ can be obtained by substituting the above results. 


\section{References}

Agarwal, A., Juneja, S., and Sircar, R. (2016). American options under stochastic volatility: control variates, maturity randomization \& multiscale asymptotics. Quantitative Finance, 16(1):17-30.

Barsotti, F. and Pontier, M. (2016). Optimal capital structure under stochastic volatility: A second order approximation. working paper, $\mathrm{xx}(\mathrm{x}): \mathrm{xxx}^{-}$ $\mathrm{xxx}$.

Brennan, M. J. (1991). The price of convenience and the valuation of commodity contingent claims. Stochastic models and option values, 200:33-71.

Cassese, G. and Guidolin, M. (2006). Modelling the implied volatility surface: Does market efficiency matter? an application to mib30 index options. International Review of Financial Analysis, 15:145-178.

Chiarella, C., Kang, B., Nikitopoulos, C., and Tô, T.-D. (2015). The returnvolatility relation in commodity futures markets. The Journal of Futures Markets, 36(2):127-152.

Fouque, J.-P., Papanicolaou, G., and Sircar, K. (2000). Stochastic volatility correction to black-scholes. Risk, 13(2):89-92.

Gatheral, J. (2011). The volatility surface: a practitioner's guide, volume 357. John Wiley \& Sons.

Gibson, R. and Schwartz, E. S. (1990). Stochastic convenience yield and the pricing of oil contingent claims. The Journal of Finance, 45(3):959-976.

Hilliard, J. E. and Reis, J. (1998). Valuation of commodity futures and options under stochastic convenience yields, interest rates, and jump diffusions in the spot. Journal of Financial and Quantitative Analysis, 33(1).

Hull, J. C. (2006). Options, futures, and other derivatives. Pearson Education India.

Miltersen, K. R. and Schwartz, E. S. (1998). Pricing of options on commodity futures with stochastic term structures of convenience yields and interest rates. Journal of Financial and Quantitative Analysis, 33(01):33-59.

Rauch, J., Krayzler, M., Brunner, B., and Zagst, R. (2013). Pricing of derivatives on commodity indices. International Review of Financial Analysis, 29:143-151. 
Schwartz, E. S. (1997). The stochastic behavior of commodity prices: Implications for valuation and hedging. The Journal of Finance, 52(3):923-973.

Ting, S. (2013). Asymptotic techniques and stochastic volatility in option pricing problem. PhD Thesis, University of Sydney, available at https://ses.library.usyd.edu.au/handle/2123/8751.

Ting, S. and Ewald, C.-O. (2013). On the performance of asymptotic locally risk minimising hedges in the heston stochastic volatility model. Quantitative Finance, 66(1):22-32.

Ting, S., Ewald, C.-O., and Wang, W.-K. (2013). On the investmentuncertainty relationship in a real option model with stochastic volatility. 13(16):939-954.

Trolle, A. and Schwartz, E. (2009). Unspanned stochastic volatility and the pricing of commodity derivatives. Review of Financial Studies, 22(11):4423-4461.

Zaevski, T. S., Kim, Y. S., and Fabozzi, F. J. (2014). Option pricing under stochastic volatility and tempered stable lévy jumps. International Review of Financial Analysis, 31:101-108. 